\title{
ABO Blood Type Has No Impact on Survival in Patients with Endometrial Carcinoma - A cohort study of 1074 patients
}

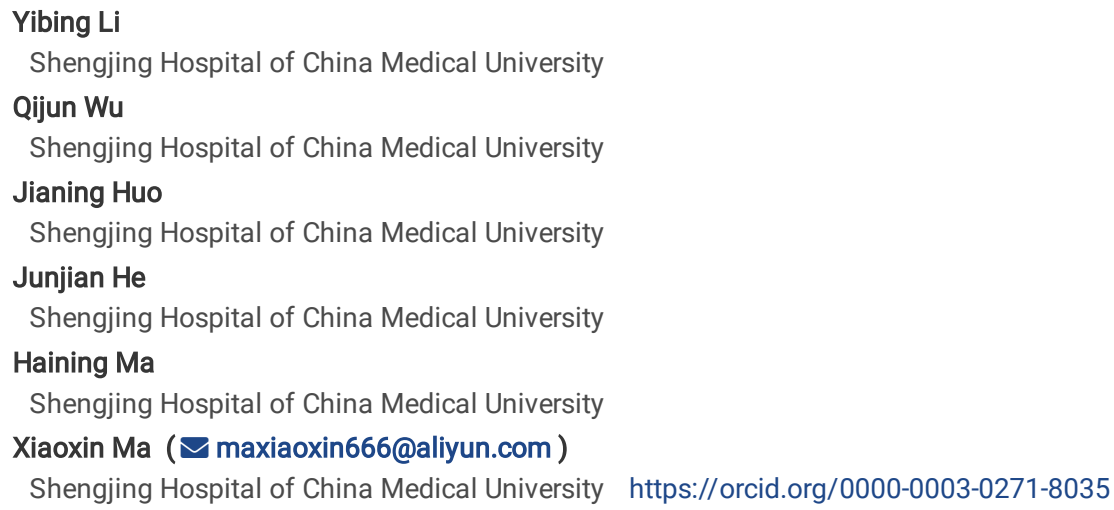




\begin{abstract}
Background: Endometrial carcinoma is one of the three major malignant tumors in gynecology. ABO blood type is associated with the prognosis of a variety of malignancies. This study assessed the relationship between $\mathrm{ABO}$ blood type and prognosis of endometrial carcinoma.
\end{abstract}

Methods: We retrospectively analyzed the relationship between ABO blood type and endometrial carcinoma prognosis in patients with primary endometrial carcinoma who underwent surgery from Shengjing Hospital affiliated to China Medical University from January 2012 to February 2017 . Univariate analysis, multivariate analysis, and stratified analysis were performed.

Results: In the multivariate analysis, $2009 \mathrm{FIGO}$ stage $(\mathrm{HR}=2.806,95 \% \mathrm{Cl}=1.289,6.109)$, Pathological tissue type $(\mathrm{HR}=0.199,95 \% \mathrm{Cl}=0.079,0.503)$ was an independent and important risk factor for OS. We divided the $\mathrm{ABO}$ blood type into $\mathrm{A}$ and non-A groups, $\mathrm{B}$ and non- $\mathrm{B}$ groups, $\mathrm{O}$ and non-O groups, $\mathrm{AB}$ and non$A B$ groups, and failed to measure the significant results of OS. After we excluded 9 patients who had recurrence, metastasis, or death within 1 year of enrollment, the OS-significant results were similar to those described above.

Conclusions: Our study suggest that there is no association between ABO blood type and EC prognosis, and additional cohort studies are needed for validation.

\title{
1. Introduction
}

Endometrial carcinoma (EC) is one of the three major malignant tumors in gynecology. In the United States, there are 61,880 new cases each year, second only to breast cancer, lung and bronchial cancer, and colorectal cancer. There are 12,160 deaths, second only to lung and bronchial cancer, breast cancer, colorectal cancer, pancreatic cancer and Ovarian cancer, ranked sixth. ${ }^{[1]}$ The most important risk factors in EC are obesity, persistent endogenous or exogenous hyperestrogen (polycystic ovary, tamoxifen treatment, anovulation and nulliparity), hypertension and diabetes. Moreover, women with Lynch syndrome (LS or hereditary non-colon cancer) have a significantly increased risk of developing EC. Most patients with EC have a good prognosis, but have a survival rate of less than $50 \%$ for patients with high-risk early stage disease or advanced diagnosis. ${ }^{2]}$

The ABO blood group system is the first blood type system discovered and determined by Landsteiner in 1900. A blood type system classified according to the presence or absence of specific antigens (aggregates) A and B on the surface of red blood cells. According to the distribution of agglutinogens $A$ and $B$, blood is divided into four types: $A, B, A B$, and 0 . Studies have shown that the risk of diffuse gastric cancer in patients with type $A$ blood is increased by $20 \%$ compared with people with type $O$ blood, ${ }^{[3]}$ people with type $A$ blood have an increased risk of breast cancer. ${ }^{[4,5]}$ In addition, studies have shown that patients with type $B$ or $A B$ blood have lower survival rates and increased risk of recurrence after breast cancer. ${ }^{[6]}$ Chang et al. showed that type $A$ blood is associated with a higher incidence and metastatic rate of malignant melanoma of the skin, ${ }^{[7]}$ blood type $B$ It also significantly reduced the risk of stomach cancer and bladder cancer, while blood type AB significantly increased the risk of liver cancer. ${ }^{[8]}$ Moreover, studies have shown that $A B O$ blood type has no effect on the survival of patients with epithelial ovarian cancer. ${ }^{[9]}$

Previous studies have shown that regardless of menopausal status, body mass index, oral contraceptive use or family cancer history, blood group A is positively associated with EC risk in Chinese women ${ }^{[10]}$. To investigate the relationship between ABO blood group and prognosis of EC, we conducted a retrospective study at Shengjing Hospital of China Medical University.

\section{Material And Methods}

\subsection{Study population}

Approved by the Institutional Review Committee (Ethical No.2017PS292K) of the Shengjing Hospital affiliated to China Medical University, we conducted a retrospective study of EC patients from January 2012 to February 2017 at Shengjing Hospital of China Medical University. Includes patients who have been diagnosed with primary EC and undergo surgery. The patient exclusion criteria were as follows: 1. Patients with other tumors at the same time; 2 . Patients undergoing treatment for recurrent disease; 3. Patients receiving chemotherapy or radiotherapy at Shengjing Hospital after surgery in other hospitals; 4. Patients under 15 years of age 5. Patients with incomplete data for variables or covariates analyzed in this study.

\subsection{Data collection}

According to the electronic medical records of the electronic information system of Shengjing Hospital affiliated to China Medical University, we collected the following demographic and clinical variables: age of diagnosis, menopause, birth history, degree of tumor differentiation, 2009 FIGO stage, Depth of infiltration(layer), lymphatic vessel interstitial infiltration, Lymph node metastasis, pathological tissue types, etc. At the same time, the blood type of the patient before the operation was obtained through the Shengjing Hospital inspection system. All data were collected by experienced gynaecologists and pathologists Tumor staging was calculated according to the 2009 International Obstetrics and Gynecology Alliance (FIGO) staging standard. The degree of differentiation of tumors is divided into high differentiation, high-medium differentiation, medium differentiation, medium-low differentiation, and low differentiation. The depth of infiltration(layer) is divided into mucosal layers, less than $1 / 2$, greater than or equal to $1 / 2$. According to the pathological tissue type, it is divided into adenocarcinoma and non-adenocarcinoma

\subsection{Follow-up and outcome}

Page $2 / 11$ 
After surgery, all enrolled patients were followed up by telephone for follow-up. The primary endpoint was overall survival (OS), which was defined as the date from completion of the procedure to the patient's death for any reason, or the date of the last follow-up of the surviving patient (2018.7), the cause of death was from telephone follow-up and proof of death.

\subsection{Statistical analysis}

The Chi-square test was used to compare continuous and categorical variables in different blood types, respectively. Age at diagnosis is summarized as means $\pm S D$. Follow-up time is summarized as the median of the inter-quartile range (IQR). The categorical variables are expressed as numbers and percentages. The Cox proportional hazard model was used to estimate risk ratios (HRs) and $95 \%$ confidence intervals (Cls). We use the likelihood ratio test to assess the proportional hazard assumption. The $A B O$ blood type is divided into four groups: $A, B, O$, and $A B$. In addition, we conducted the following four subgroups, group $A$ and non-group $A$, group $B$ and non-group $B$, group $O$ and non-group 0 , group $A B$ and non-group $A B$.

At the same time, we performed Multivariable adjusted analyses, including the following potential confounders: age at diagnosis, menopause, birth history, degree of tumor differentiation, FIGO stage, Depth of infiltration(layer), lymphatic vessel infiltration, Lymph node metastasis, pathological tissue type. In addition, we conducted a subgroup analysis of stratification of potential confounding factors by appeal. A likelihood ratio test was performed to check whether the association between ABO blood group and OS was modified by the following pre-specified potential effect modifiers: menopause, birth history, degree of tumor differentiation, FIGO stage, Depth of infiltration(layer), lymphatic vessels Interstitial infiltration, Lymph node metastasis, pathological tissue type. We further excluded by sensitivity analysis: patients with recurrence, metastasis, or patients who died within 1 year of study enrollment. P value $<0.05$ was considered statistically significant. All analyses were performed using the IBM SPSS Statistics 24 software.

\section{Results}

\subsection{Patient characteristics}

As shown in Figure 1, information of variables and covariates were incomplete after the exclusion, a total of 1074 patients were included in the study. Among them, 17 patients had recurrence, 30 patients had metastasis, and 40 patients died. The demographic and clinical characteristics of patients with EC according to $A B O$ blood type are shown in Table 1. The median age of blood type A, B, O, AB was $56.14( \pm 8.38), 56.19( \pm 9.00), 56.02( \pm 8.92), 55.54( \pm 9.11)$. Blood type $A, B, O$ and $A B$ accounted for $28.6 \%, 34.0 \%, 26.7 \%$ and $10.7 \%$, respectively. There were no significant differences in $A B O$ blood type in terms of demographics and clinical characteristics. 
Table 1

Selected demographic and clinical characteristics of Endometrial carcinoma patients according to ABO blood type

\begin{tabular}{|c|c|c|c|c|c|}
\hline \multirow[t]{2}{*}{ Variables } & \multicolumn{4}{|l|}{ ABO blood type } & \multirow[t]{2}{*}{$P$ value } \\
\hline & 0 & $A$ & B & $A B$ & \\
\hline Total case & 287 & 307 & 365 & 115 & \\
\hline Age at diagnosis(years) & $56.02 \pm 8.92$ & $56.14 \pm 8.38$ & $56.19 \pm 9.00$ & $55.54 \pm 9.11$ & 0.917 \\
\hline Follow-up time(years) & $3.66(2.29 \sim 4.59)$ & $3.94(2.58 \sim 4.98)$ & $3.89(2.50 \sim 5.04)$ & $3.53(2.52 \sim 4.84)$ & 0.057 \\
\hline Vital status(\%) & 287 & 307 & 365 & 115 & 0.656 \\
\hline Alive & $273(95.1)$ & $296 \rrbracket 96.4 \rrbracket$ & $354(97.0)$ & $111(96.5)$ & \\
\hline Died & $14(4.9)$ & $11 \rrbracket 3.6 \rrbracket$ & $11(3.0)$ & $4(3.5)$ & \\
\hline Recurrence status & & & & & 0.120 \\
\hline Yes & 8 & 6 & 2 & 1 & \\
\hline No & 279 & 301 & 363 & 114 & \\
\hline Metastasis & & & & & 0.230 \\
\hline Yes & 11 & 10 & 5 & 4 & \\
\hline No & 276 & 297 & 360 & 111 & \\
\hline Menopause & & & & & 0.672 \\
\hline Yes & 95 & 216 & 241 & 37 & \\
\hline No & 192 & 91 & 124 & 78 & \\
\hline Birth history & & & & & 0.787 \\
\hline Yes & 270 & 289 & 347 & 107 & \\
\hline No & 17 & 20 & 18 & 8 & \\
\hline Degree of tumor differentiation & & & & & 0.717 \\
\hline High differentiation & 132 & 136 & 156 & 51 & \\
\hline High-medium differentiation & 44 & 45 & 46 & 14 & \\
\hline Medium differentiation & 65 & 75 & 89 & 33 & \\
\hline Medium-low differentiation & 21 & 28 & 32 & 5 & \\
\hline Low differentiation & 25 & 23 & 42 & 12 & \\
\hline 2009 FIGO stage & & & & & 0.477 \\
\hline Stage I & 235 & 253 & 286 & 90 & \\
\hline Stage II, III, IV & 52 & 54 & 79 & 25 & \\
\hline Depth of infiltration(layer) & & & & & 0.703 \\
\hline Mucosal layer & 54 & 60 & 61 & 25 & \\
\hline$\nabla 1 / 2$ & 171 & 168 & 216 & 61 & \\
\hline$\geq 1 / 2$ & 62 & 79 & 88 & 29 & \\
\hline Lymphatic vessels Interstitial infiltration & & & & & 0.625 \\
\hline Yes & 263 & 285 & 330 & 103 & \\
\hline No & 24 & 22 & 35 & 12 & \\
\hline Lymph node metastasis & & & & & 0.587 \\
\hline Yes & 266 & 288 & 334 & 104 & \\
\hline No & 21 & 19 & 31 & 11 & \\
\hline Pathological tissue type & & & & & 0.390 \\
\hline Adenocarcinoma & 6 & 9 & 15 & 2 & \\
\hline Non-adenocarcinoma & 281 & 298 & 350 & 113 & \\
\hline
\end{tabular}




\subsection{Multivariate analyses of the demographic and clinical characteristics}

Table 2 summarizes the adjusted OS-related patient characteristics. In the multivariate analysis, $2009 \mathrm{FIGO}$ stage (HR=2.806, 95\% $\mathrm{Cl}=1.289,6.109)$, Pathological tissue type ( $\mathrm{HR}=0.199,95 \% \mathrm{Cl}=0.079,0.503)$ was an independent and important risk factor for poor OS. Age, Menopause, birth history, Degree of tumor differentiation, Depth of infiltration (layer), Lymphatic vessels Interstitial infiltration, Lymph node metastasis is not significantly associated with OS.

Table 2

Selected demographic and clinical characteristics according to overall survival among endometrial carcinoma patients

\begin{tabular}{|c|c|c|c|}
\hline \multirow[t]{2}{*}{ Variables } & \multicolumn{2}{|l|}{ OS } & \multirow[t]{2}{*}{$P$ value } \\
\hline & No./Events & $\mathrm{HR}(95 \% \mathrm{Cl}) \dagger$ & \\
\hline \multicolumn{4}{|l|}{ Age at diagnosis(years) } \\
\hline$\leq 55$ & $468 / 11$ & Reference & \\
\hline$\varangle 55$ & $606 / 29$ & 1.671(0.739ه3.775) & 0.217 \\
\hline \multicolumn{4}{|l|}{ Menopause } \\
\hline No & $347 / 7$ & Reference & \\
\hline Yes & $727 / 33$ & $1.256 \rrbracket 0.482,3.275 \rrbracket$ & 0.641 \\
\hline \multicolumn{4}{|l|}{ Birth history } \\
\hline No & $63 / 1$ & Reference & \\
\hline Yes & $1011 / 39$ & $2.103(0.280,15.782)$ & 0.470 \\
\hline Degree of tumor differentiation & & & 0.737 \\
\hline High differentiation & $475 / 9$ & Reference & \\
\hline High-medium differentiation & $149 / 5$ & $1.252(0.414,3.788)$ & 0.691 \\
\hline Medium differentiation & $262 / 12$ & $1.499(0.616,3.653)$ & 0.372 \\
\hline Medium-low differentiation & $86 / 7$ & $1.881(0.648,5.454)$ & 0.245 \\
\hline Low differentiation & $102 / 7$ & $1.923(0.665,5.562)$ & 0.228 \\
\hline \multicolumn{4}{|l|}{2009 FIGO stage } \\
\hline Stage I & $864 / 21$ & Reference & \\
\hline Stage II, III, IV & $210 / 19$ & $2.806(1.289,6.109)$ & 0.009 \\
\hline Depth of infiltration(layer) (\%) & & & 0.120 \\
\hline Mucosal layer & $200 / 3$ & Reference & \\
\hline$\otimes 1 / 2$ & $616 / 19$ & $2.184(0.632,7.550)$ & 0.217 \\
\hline$\geq 1 / 2$ & $258 / 18$ & $3.571(0.983 .12 .979)$ & 0.053 \\
\hline \multicolumn{4}{|c|}{ Lymphatic vessels Interstitial infiltration } \\
\hline No & $981 / 32$ & Reference & \\
\hline Yes & $93 / 8$ & $0.251(0.033,1.895)$ & 0.180 \\
\hline \multicolumn{4}{|l|}{ Lymph node metastasis } \\
\hline No & $992 / 31$ & Reference & \\
\hline Yes & $82 / 9$ & $3.057(0.406,23.021)$ & 0.278 \\
\hline \multicolumn{4}{|l|}{ Pathological tissue type } \\
\hline No & $32 / 7$ & Reference & \\
\hline Yes & $1042 / 33$ & $0.199(0.079,0.503)$ & 0.001 \\
\hline
\end{tabular}

\subsection{Association of ABO blood type and survival of EC patients}

Multivariate analysis in table 3 suggested that Pathological tissue type was an independent risk factor for OS ( $\mathrm{HR}=0.206,95 \% \mathrm{Cl}=0.080,0.533)$, but no other variables including $A B O$ blood group were significantly associated. We grouped $A B O$ blood type into blood type $A$ and blood type non- $A$, blood type $B$ and blood type non-B, blood type $O$ and blood type non-O, blood type $A B$ and blood type non-AB. And we failed to measure the significant results of OS. (Table 4-5). 
At the same time, we excluded the 9 patients who had recurrence, metastasis or death within 1 year, and the OS significant results were similar to the above results.

Table 3

Prognostic factors for overall survival by multivariate analysis.

\begin{tabular}{|c|c|c|c|}
\hline \multirow[t]{2}{*}{ Variables } & \multicolumn{2}{|l|}{ os } & \multirow[t]{2}{*}{$P$ value } \\
\hline & HR & $95 \% \mathrm{Cl}$ & \\
\hline \multicolumn{4}{|l|}{ Menopause } \\
\hline No & Reference & - & \\
\hline Yes & 1.630 & $0.696,3.819$ & 0.260 \\
\hline \multicolumn{4}{|l|}{ Birth history } \\
\hline No & Reference & - & \\
\hline Yes & 2.061 & $0.275,15.446$ & 0.482 \\
\hline Degree of tumor differentiation & & & 0.746 \\
\hline High differentiation & Reference & - & \\
\hline High-medium differentiation & 1.261 & $0.416,3.821$ & 0.682 \\
\hline Medium differentiation & 1.517 & $0.624,3.689$ & 0.358 \\
\hline Medium-low differentiation & 1.963 & $0.676,5.702$ & 0.215 \\
\hline Low differentiation & 1.816 & $0.622,5.299$ & 0.275 \\
\hline \multicolumn{4}{|l|}{2009 FIGO stage } \\
\hline Stage I & Reference & - & \\
\hline Stage II, III, IV & 2.519 & $1.144,5.548$ & 0.022 \\
\hline Depth of infiltration(layer) (\%) & & & 0.099 \\
\hline Mucosal layer & Reference & - & \\
\hline$₫ 1 / 2$ & 2.014 & $0.588,6.897$ & 0.265 \\
\hline$\geq 1 / 2$ & 3.618 & $0.992,13.199$ & 0.051 \\
\hline \multicolumn{4}{|c|}{ Lymphatic vessels Interstitial infiltration } \\
\hline No & Reference & - & \\
\hline Yes & 0.321 & $0.044,2.335$ & 0.261 \\
\hline \multicolumn{4}{|l|}{ Lymph node metastasis } \\
\hline No & Reference & - & \\
\hline Yes & 2.517 & $0.348,18.185$ & 0.360 \\
\hline \multicolumn{4}{|l|}{ Pathological tissue type } \\
\hline No & Reference & - & \\
\hline Yes & 0.206 & $0.080,0.533$ & 0.001 \\
\hline ABO blood type & & & 0.567 \\
\hline Type A & Reference & - & \\
\hline Type B & 0.687 & $0.220,2.147$ & 0.519 \\
\hline Type 0 & 0.638 & $0.282,1.446$ & 0.278 \\
\hline Type AB & 0.582 & $0.259,1.305$ & 0.189 \\
\hline
\end{tabular}


Table 4

Hazard ratio $(95 \% \mathrm{Cl})$ for overall survival among endometrial carcinoma patients according to $\mathrm{A}$ and non-A blood type, $\mathrm{B}$ and non-B blood ty $\mathrm{F}$

\begin{tabular}{|c|c|c|c|c|c|c|c|c|}
\hline \multirow[t]{3}{*}{ Variables } & \multicolumn{2}{|l|}{ Overall survival } & \multirow{3}{*}{$\begin{array}{l}P \\
\text { value }\end{array}$} & \multicolumn{2}{|l|}{ Overall survival } & \multirow{3}{*}{$\begin{array}{l}P \\
\text { value }\end{array}$} & \multicolumn{2}{|l|}{ Overall survival } \\
\hline & Blood type A & $\begin{array}{l}\text { Blood type } \\
\text { non-A }\end{array}$ & & Blood type B & $\begin{array}{l}\text { Blood type } \\
\text { non-B }\end{array}$ & & Blood type 0 & $\begin{array}{l}\mathrm{Bl} \\
\mathrm{ne}\end{array}$ \\
\hline & $\mathrm{HR}(95 \% \mathrm{Cl})$ & $\mathrm{HR}(95 \% \mathrm{Cl})$ & & $\mathrm{HR}(95 \% \mathrm{Cl})$ & $\mathrm{HR}(95 \% \mathrm{Cl})$ & & $\mathrm{HR}(95 \% \mathrm{Cl})$ & $\mathrm{H}$ \\
\hline \multicolumn{9}{|l|}{ All patients } \\
\hline Menopause & & & 0.252 & & & 0.931 & & \\
\hline No & $1.969(0.440,8.800)$ & Reference & & $0.683(0.132,3.523)$ & Reference & & $0.471(0.057,3.919)$ & $\mathrm{Re}_{\mathrm{f}}$ \\
\hline Yes & $0.734(0.331,1.628)$ & Reference & & $0.746(0.347,1.605)$ & Reference & & $1.920(0.955,3.861)$ & Rt \\
\hline Birth history & & & 0.968 & & & 0.972 & & \\
\hline No & $198.074(0.000,3.345 E+10)$ & Reference & & $0.030(0.000,3035495.32)$ & Reference & & $0.030(0.000,3035495.32)$ & Rt \\
\hline Yes & $0.836(0.407,1.716)$ & Reference & & $0.733(0.365,1.472)$ & Reference & & $1.629(0.847,3.134)$ & $\mathrm{Rt}$ \\
\hline $\begin{array}{l}\text { Degree of tumor } \\
\text { differentiation }\end{array}$ & & & 0.573 & & & 0.986 & & \\
\hline $\begin{array}{l}\text { High } \\
\text { differentiation }\end{array}$ & $0.660(0.137,3.180)$ & Reference & & $0.567(0.118,2.729)$ & Reference & & $2.284(0.613,8.514)$ & $\mathrm{Rt}$ \\
\hline $\begin{array}{l}\text { High-medium } \\
\text { differentiation }\end{array}$ & $3.341(0.558,20.010)$ & Reference & & $0.536(0.060,4.794)$ & Reference & & $0.628(0.070,5.619)$ & $\mathrm{Rt}$ \\
\hline $\begin{array}{l}\text { Medium } \\
\text { differentiation }\end{array}$ & $0.542(0.119,2.474)$ & Reference & & $0.894(0.269,2.972)$ & Reference & & $1.542(0.464,5.122)$ & $\mathrm{Re}$ \\
\hline $\begin{array}{l}\text { Medium-low } \\
\text { differentiation }\end{array}$ & $0.904(0.175,4.681)$ & Reference & & $0.696(0.134,3.623)$ & Reference & & $2.093(0.447,9.804)$ & $\mathrm{Rt}$ \\
\hline $\begin{array}{l}\text { Low } \\
\text { differentiation }\end{array}$ & $1.199(0.230,6.233)$ & Reference & & $0.548(0.106,2.833)$ & Reference & & $1.343(0.259,6.972)$ & $\mathrm{Rt}$ \\
\hline $\begin{array}{l}2009 \text { FIGO } \\
\text { stage }\end{array}$ & & & 0.454 & & & 0.265 & & \\
\hline Stage I & $1.188(0.480,2.944)$ & Reference & & $0.448(0.151,1.333)$ & Reference & & $1.419(0.572,3.519)$ & Rt \\
\hline Stage II, III, IV & $0.725(0.241,2.186)$ & Reference & & $1.003(0.395,2.547)$ & Reference & & $1.858(0.731,4.719)$ & Rt \\
\hline $\begin{array}{l}\text { Depth of } \\
\text { infiltration(layer) } \\
(\%)\end{array}$ & & & 0.685 & & & 0.933 & & \\
\hline Mucosal layer & $1.129(0.102,12.451)$ & Reference & & $1.120(0.102,12.352)$ & Reference & & $1.386(0.126,15.286)$ & Rt \\
\hline$\otimes 1 / 2$ & $1.171(0.445,3.083)$ & Reference & & $0.670(0.241,1.861)$ & Reference & & $1.972(0.793,4.904)$ & $\mathrm{Rt}$ \\
\hline$\geq 1 / 2$ & $0.633(0.208,1.922)$ & Reference & & $0.682(0.243,1.918)$ & Reference & & $1.346(0.479,3.779)$ & $\mathrm{Rt}$ \\
\hline $\begin{array}{l}\text { Lymphatic } \\
\text { vessels } \\
\text { Interstitial } \\
\text { infiltration }\end{array}$ & & & 0.366 & & & 0.181 & & \\
\hline No & $1.080(0.511,2.280)$ & Reference & & $0.540(0.233,1.248)$ & Reference & & $1.496(0.721,3.103)$ & $\mathrm{Rt}$ \\
\hline Yes & $0.424(0.052,3.447)$ & Reference & & $1.629(0.407,6.514)$ & Reference & & $1.892(0.452,7.925)$ & $\mathrm{Rt}$ \\
\hline $\begin{array}{l}\text { Lymph node } \\
\text { metastasis }\end{array}$ & & & 0.278 & & & 0.306 & & \\
\hline No & $1.134(0.534,2.408)$ & Reference & & $0.562(0.242,1.305)$ & Reference & & $1.352(0.636,2.871)$ & $\mathrm{Rt}$ \\
\hline Yes & $0.363(0.045,2.903)$ & Reference & & $1.264(0.339,4.709)$ & Reference & & $2.828(0.757,10.569)$ & Rt \\
\hline $\begin{array}{l}\text { Pathological } \\
\text { tissue type }\end{array}$ & & & 0.935 & & & 0.654 & & \\
\hline No & $0.873(0.169,4.506)$ & Reference & & $0.523(0.101,2.696)$ & Reference & & $3.514(0.785,15.724)$ & Rt \\
\hline Yes & $0.901(0.419,1.939)$ & Reference & & $0.716(0.333,1.540)$ & Reference & & $1.439(0.698,2.969)$ & $\mathrm{R}_{t}$ \\
\hline
\end{tabular}


Table 5

Hazard ratio $(95 \% \mathrm{Cl})$ for overall survival among endometrial carcinoma patients according to $\mathrm{A}$ and non-A blood type, $\mathrm{B}$ and non-B blood type, $\mathrm{O}$ and no

\begin{tabular}{|c|c|c|c|c|c|c|c|c|}
\hline \multirow[t]{3}{*}{ Variables } & \multicolumn{2}{|l|}{ Overall survival } & \multirow{3}{*}{$\begin{array}{l}P \\
\text { value }\end{array}$} & \multicolumn{2}{|l|}{ Overall survival } & \multirow{3}{*}{$\begin{array}{l}P \\
\text { value }\end{array}$} & \multicolumn{2}{|l|}{ Overall survival } \\
\hline & Blood type A & $\begin{array}{l}\text { Blood type } \\
\text { non-A }\end{array}$ & & Blood type B & $\begin{array}{l}\text { Blood type } \\
\text { non-B }\end{array}$ & & Blood type 0 & $\begin{array}{l}\mathrm{E} \\
\mathrm{n}\end{array}$ \\
\hline & $\mathrm{HR}(95 \% \mathrm{Cl})$ & $\mathrm{HR}(95 \% \mathrm{Cl})$ & & $\mathrm{HR}(95 \% \mathrm{Cl})$ & $\mathrm{HR}(95 \% \mathrm{Cl})$ & & $\mathrm{HR}(95 \% \mathrm{Cl})$ & $r$ \\
\hline \multicolumn{9}{|l|}{ All patients } \\
\hline Menopause & & & 0.130 & & & 0.651 & & \\
\hline No & $3.834(0.641,22.953)$ & Reference & & $0.418(0.047,3.738)$ & Reference & & $0.727(0.081,6.513)$ & $\mathrm{R}$ \\
\hline Yes & $0.839(0.353,1.997)$ & Reference & & $0.731(0.307,1.739)$ & Reference & & $1.584(0.706,3.555)$ & $\mathrm{R}$ \\
\hline Birth history & & & 0.957 & & & 0.974 & & \\
\hline No & $198.074(0.000,3.345 E+10)$ & Reference & & $0.030(0.000,3035495.32)$ & Reference & & $0.030(0.000,3035495.32)$ & $\mathrm{R}$ \\
\hline Yes & $1.028(0.471,2.245)$ & Reference & & $0.673(0.300,1.513)$ & Reference & & $1.479(0.692,3.160)$ & $\mathrm{R}$ \\
\hline $\begin{array}{l}\text { Degree of tumor } \\
\text { differentiation }\end{array}$ & & & 0.802 & & & 0.982 & & \\
\hline $\begin{array}{l}\text { High } \\
\text { differentiation }\end{array}$ & $0.763(0.154,3.784)$ & Reference & & $0.659(0.133,3.265)$ & Reference & & $1.733(0.414,7.257)$ & $\mathrm{R}$ \\
\hline $\begin{array}{l}\text { High-medium } \\
\text { differentiation }\end{array}$ & $2.206(0.310,15.675)$ & Reference & & $0.706(0.073,6.795)$ & Reference & & $0.848(0.088,8.156)$ & $\mathrm{R}$ \\
\hline $\begin{array}{l}\text { Medium } \\
\text { differentiation }\end{array}$ & $0.542(0.119,2.474)$ & Reference & & $0.894(0.269,2.972)$ & Reference & & $1.542(0.464,5.122)$ & $\mathrm{R}$ \\
\hline $\begin{array}{l}\text { Medium-low } \\
\text { differentiation }\end{array}$ & $1.574(0.261,9.495)$ & Reference & & $0.439(0.048,3.983)$ & Reference & & $1.742(0.268,11.313)$ & $\mathrm{R}$ \\
\hline $\begin{array}{l}\text { Low } \\
\text { differentiation }\end{array}$ & $239.745(0.000,267471512)$ & Reference & & $0.018(0.000,1837.883)$ & Reference & & $0.035(0.000,130692.197)$ & $\mathrm{R}$ \\
\hline $\begin{array}{l}2009 \text { FIGO } \\
\text { stage }\end{array}$ & & & 0.278 & & & 0.294 & & \\
\hline Stage I & $1.658(0.631,4.357)$ & Reference & & $0.403(0.116,1.403)$ & Reference & & $1.198(0.422,3.405)$ & $\mathrm{R}$ \\
\hline Stage II, III, IV & $0.727(0.203,2.606)$ & Reference & & $0.966(0.324,2.884)$ & Reference & & $1.797(0.602,5.362)$ & $\mathrm{R}$ \\
\hline $\begin{array}{l}\text { Depth of } \\
\text { infiltration(layer) } \\
\text { (\%) }\end{array}$ & & & 0.946 & & & 0.772 & & \\
\hline Mucosal layer & $1.129(0.102,12.451)$ & Reference & & $1.120(0.102,12.352)$ & Reference & & $1.386(0.126,15.286)$ & $\mathrm{R}$ \\
\hline$\otimes 1 / 2$ & $1.253(0.428,3.668)$ & Reference & & $0.470(0.133,1.667)$ & Reference & & $2.399(0.870,6.617)$ & $\mathrm{R}$ \\
\hline$\geq 1 / 2$ & $0.979(0.302,3.181)$ & Reference & & $0.767(0.236,2.498)$ & Reference & & $0.658(0.146,2.973)$ & $\mathrm{R}$ \\
\hline $\begin{array}{l}\text { Lymphatic } \\
\text { vessels } \\
\text { Interstitial } \\
\text { infiltration }\end{array}$ & & & 0.584 & & & 0.106 & & \\
\hline No & $1.250(0.557,2.805)$ & Reference & & $0.456(0.172,1.211)$ & Reference & & $1.527(0.681,3.427)$ & $\mathrm{R}$ \\
\hline Yes & $0.711(0.079,6.364)$ & Reference & & $2.435(0.407,14.577)$ & Reference & & $0.820(0.092,7.336)$ & $\mathrm{R}$ \\
\hline $\begin{array}{l}\text { Lymph node } \\
\text { metastasis }\end{array}$ & & & 0.410 & & & 0.213 & & \\
\hline No & $1.332(0.589,3.014)$ & Reference & & $0.479(0.180,1.277)$ & Reference & & $1.348(0.582,3.125)$ & $\mathrm{R}$ \\
\hline Yes & $0.548(0.064,4.696)$ & Reference & & $1.559(0.315,7.728)$ & Reference & & $1.918(0.351,10.497)$ & $\mathrm{R}$ \\
\hline $\begin{array}{l}\text { Pathological } \\
\text { tissue type }\end{array}$ & & & 0.935 & & & 0.335 & & \\
\hline No & $1.064(0.195,5.816)$ & Reference & & $0.268(0.031,2.292)$ & Reference & & $4.750(0.957,23.574)$ & $\mathrm{R}$ \\
\hline Yes & $1.118(0.482,2.592)$ & Reference & & $0.735(0.307,1.760)$ & Reference & & $1.140(0.476,2.730)$ & $\mathrm{R}$ \\
\hline
\end{tabular}

\section{Discussion}


The ABO blood type is the most important blood type system in the human blood group system. The ABO gene is located on chromosome 9 q34 and encodes two alleles of specific glycosyltransferases, namely $A$ and $B .{ }^{[11]}$ The blood group antigen is a secondary gene product, and the primary gene product is a various glycosyltransferase that binds a sugar molecule to an oligosaccharide chain. ${ }^{[12]}$

The ABO gene consists of 7 exons of more than 20KB of genomic DNA, and two key single base substitutions in the final coding exon result in amino acid substitutions, which result in a donor between A-transferase and B-transferase. The difference in nucleotide sugar substrate specificity. ${ }^{[13]}$ The blood group 0 does not have the glycosyltransferase encoded by the A and B genes, and expresses a fucosylated variant (Ley) of the precursor structure. ${ }^{[14]}$ Studies have shown that $\mathrm{ABO}$ blood group plays an important role in affecting circulating SP-selectin and sICAM-1 levels, which may be related to glycosylation of $\mathrm{P}$ selectin/ICAM-1 from cell membrane detachment interaction. ${ }^{[15]}$

At present, multiple studies have shown that $A B O$ blood group is associated with tumor prognosis. Cao et al ${ }^{[16]}$ showed that Blood type $A B$ is a favourable prognostic factor for patients with colon cancer. Studies by DONATAS STAKIŠAITIS have shown that type B blood is associated with prostate cancer and bladder cancer risk and can be assessed as a determinant of negative longevity. ${ }^{[17]}$ A study by Ting Jin et al ${ }^{[18]}$ showed that the $0 S$ of patients with blood group $O$ was significantly shorter than that of other $A B O$ patients. In addition, prospective studies have shown that type B and type $A B$ blood are associated with a significant reduction in the risk of gastrointestinal and colorectal cancer, respectively, and type B blood reduces the risk of stomach cancer and bladder cancer, and type AB blood increases liver cancer. The risk, but not related to the risk of sarcoma, lymphoma, leukemia or other cell types of cancer. ${ }^{[8]} \mathrm{A}$ retrospective study by $\mathrm{Li}$ et al showed that there is an association between the ABO blood types and the survival of Chinese patients with resected NSCLC. And the overall survival, patients-free survival and locoregional relapse-free survival were significantly prolonged in patients with a blood group of $O$ or $B$ compared with patients with blood group $A$ or $A B{ }^{[19]}$

There are currently few studies on ABO blood type and EC. A retrospective cohort study of 203 patients with type I EC found that patients with type A blood had a lower risk of developing G3 tumors than patients with non-type A blood. ${ }^{[20]}$ Type A blood is associated with a high risk of EC ${ }^{[21]}$, whereas conversely, A

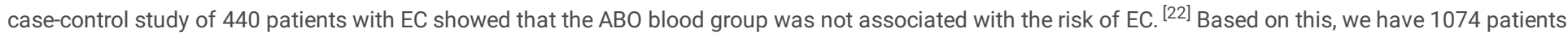
from Shengjing Hospital affiliated to China Medical University from January 2012 to February 2017. A retrospective study was conducted and a large number of subgroup analyses were performed. Multivariate analysis showed that histological type was an independent and important risk factor for OS, while other factors were not significantly associated with OS. At the same time, we divided the ABO blood type into A group and non-A group, B group and non-B group, $O$ group and non-O group, $A B$ group and non-AB group respectively for stratified analysis, no significant interaction, further we Sensitivity analysis was performed on patients who had relapsed, metastasized, and died within 1 year of enrollment. OS outcomes were similar. For our study, the sample size was larger compared to previous studies related to ABO blood group and EC. Moreover, our sample information comes from Shengjing Hospital affiliated to China Medical University, and its electronic medical record system is perfect, thereby minimizing the likelihood of recall bias. On the other hand, our study had a short follow-up period (median follow-up period was year), and the number of missing dependent variables and covariates was 1032 . However, we found no difference between the patients included and excluded.

\section{Conclusion}

In summary, our data suggest that there is no association between ABO blood type and EC prognosis, and additional cohort studies are needed to validate.

\section{Abbreviations}

EC: endometrial cancer

FIGO: International Federation of Gynecology and Obstetrics

OS: overall survival

IQR: inter-quartile range

HR: Hazard Ratio

NSCLC: non-small cell lung cancer

\section{Declarations}

\section{Ethics approval and consent to participate}

Our study is approved by the Institutional Review Committee of the Ethics Committee of Shengjing Hospital of China Medical University (No.2017PS292K), and it conforms to the provisions of the Declaration of Helsinki.

\section{Funding}

Our study was supported by the National Natural Science Foundation of China (No. 81872123), Liaoning Provincial Higher Education Innovation Team, Distinguished Professor of Liaoning Province, China Medical University's 2018 Discipline Construction “Major Special Construction Plan” (No. 3110118029) 
and Outstanding Scientific Fund of Shengjing Hospital (No. 201601). The funding body had no involvement in the design of the study, collection, analysis, interpretation of data or in writing the manuscript.

\section{Author contributions}

All authors contributed toward data analysis, drafting and critically revising the paper and agree to be accountable for all aspects of the work.

\section{Conflict of interest}

The authors declare no conflict of interest.

\section{Acknowledgements}

We are grateful for the National Natural Science Foundation of China, Liaoning Provincial Higher Education Innovation Team, Distinguished Professor of Liaoning Province, China Medical University's 2018 Discipline Construction "Major Special Construction Plan" and Outstanding Scientific Fund of Shengjing Hospital.

\section{Reference}

[1] Rebecca L. Siegel, Kimberly D. Miller, Ahmedin Jemal. cancer statistics, 2019. CA CANCER J CLIN. 2019;69:7-34

[2] A. Santaballa, X. Matías-Guiu, A. Redondo, N. Carballo, M. Gil, C. Gómez, M. Gorostidi, M. Gutierrez, A. Gónzalez-Martín. SEOM clinical guidelines for endometrial cancer (2017) , Clin Transl Oncol. 2018;04;20(4):559-560

[3]Edgren G, Hjalgrim H, Rostgaard K, Norda R, Wikman A, Melbye M, Nyrén O. Risk of gastric cancer and peptic ulcers in relation To ABO blood type: A cohort study. Am J Epidemiol. 2010; 172(11): 1280-85

[4]Zhang BL, He N, Huang YB, Song FJ, Chen KX. ABO blood groups and risk of cancer: A systematic review and meta-analysis. Asian Pac J Cancer Prev, 2014; 15(11): 4643-50

[5] Miao SY, Zhou W, Chen L, Wang S, Liu XA. Influence of ABO blood group and Rhesus factor on breast cancer risk: A meta-analysis of 9,665 breast cancer patients and 244,768 controls. Asia Pac J Clin Oncol, 2014; 10(2): 101-8

[6] Klimant E, Glurich I, Mukesh B, Onitilo AA. Blood type, hormone receptor status, HER2/neu status, and survival in breast cancer: A retrospective study exploring relationships in a phenotypically well-defined cohort. Clin Med Res. 2011;9(3-4): 111-18

[7]Chang L, Pei J, Li C, Zhang P, Zhou D, Du W, Liu X, Jiang C. Incidence and Metastasis of Cutaneous Malignant Melanoma with Respect to ABO Blood Groups: A Case-controlled Study in Northeast of China. PLoS ONE. 2014;9(2):e88096

[8]Huang JY, Wang R, Gao YT, Yuan JM. ABO blood type and the risk of cancer - Findings from the Shanghai Cohort Study. PLoS ONE. 2017;12(9):e0184295

[9]Wang L, Yang Z, Liu Y, Wang YN, Guo JY, Wu QJ, Gong TT. ABO Blood Type Has No Impact on Survival in Patients with Epithelial Ovarian Cancer. J Cancer. 2018;9(23):4334-4340

[10]Xu WH, Zheng W, Xiang YB, Shu XO. ABO blood type is associated with endometrial cancer risk in Chinese women. Chin J Cancer. 2011 Nov;30(11):766771

[11]Massimo Franchini, Giancarlo M. Liumbruno, Giuseppe Lippi. The prognostic value of ABO blood group in cancer patients. Blood Transfus. 2016;14(5):434-440

[12] D Rose Ewald, Susan CJ Sumner. Blood Type Biochemistry and Human Disease. Wiley Interdiscip Rev Syst Biol Med. 2016; 8(6): 517-535

[13] Yamamoto F. (2000) Molecular genetics of ABO. Vox Sang. 78, 91-103

[14] Dabelsteen E. ABO blood group antigens in oral mucosa. What is new? J Oral Pathol Med. 2002;31(2): 65-70

[15] Barbalic M, Dupuis J, Dehghan A, Bis JC, Hoogeveen RC, Schnabel RB, Nambi V, Bretler M, Smith NL, Peters A, Lu C, Tracy RP, Aleksic N, Heeriga J, Keaney JF, Rice K, Lip GY, Vasan RS, Glazer NL, Larson MG, Uitterlinden AG, Yamamoto J, Durda P, Haritunians T, Psaty BM, Boerwinkle E, Hofman A, Koenig W, Jenny NS, Witteman JC, Ballantyne C, Benjamin EJ. Large-scale genomic studies reveal central role of ABO in sP-selectin and sICAM-1 levels. Hum. Mol. Genet. 2010;19(9):1863-1872

[16] Cao X, Wen ZS, Sun YJ, Li Y, Zhang L, Han YJ. Prognostic value of ABO blood group in patients with surgically resected colon cancer. Br. J. Cancer 2014;111(1): 174-180 
[17] Stakišaitis D, Juknevičienè M, Ulys A, Žaliūnienè D, Stanislovaitienė $D$, Šepetienè R, Slavinska A, Sužiedèlis $K$, Lesauskaitè V. ABO blood group polymorphism has an impact on prostate, kidney and bladder cancer in association with longevity. Oncol Lett. 2018;16(1):1321-1331

[18] Jin T, Li PJ, Chen XZ, Hu WH. ABO blood group is a predictor of survival in patients with laryngeal cancer. Chin J Cancer. 2016;35(1):90

[19] Li N, Xu M, Li CF, Ou W, Wang BX, Zhang SL, Xu PF, Yuan C, Huang QA, Wang SY. Prognostic role of the ABO blood types in Chinese patients with curatively resected non-small cell lung cancer: a retrospective analysis of 1601 cases at a single cancer center. Chin J Cancer. 2015;34(10):54

[20]Mandato VD, Torricelli F, Mastrofilippo V, Ciarlini G, Pirillo D, Farnetti E, Fornaciari L, Casali B, Gelli MC, Abrate M, Aguzzoli L, La Sala GB, Nicoli D. Prognostic Impact of ABO Blood Group on Type I Endometrial Cancer Patients- Results from Our Own and Other Studies. J Cancer. 2017;8(14):2828-2835

[21] Marinaccio M, Traversa A, Carioggia E, Valentino L, Coviello M, Salamanna S, Dragone DC, Marinaccio L. Blood groups of the ABO system and survival rate in gynecologic tumors. Minerva Ginecol. 1995; 47: 69-76.

[22] Yuzhalin AE, Kutikhin AG. ABO and Rh Blood Groups in Relation to Ovarian, Endometrial and Cervical Cancer Risk Among The Population of South-East Siberia. Asian Pac. J. Cancer Prev. 2012;13(10)®5091-5096

\section{Figures}

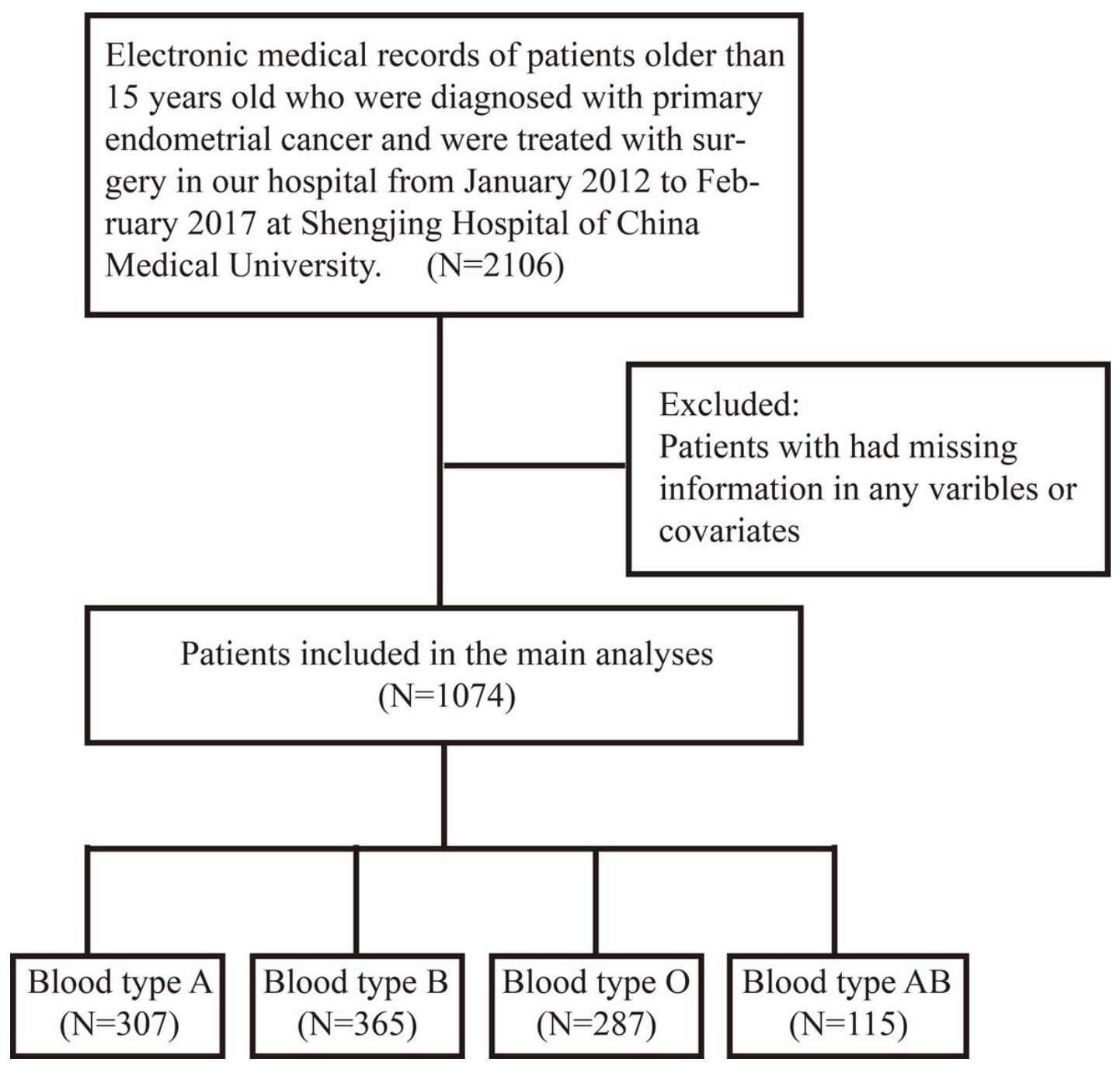

Figure 1

Flow diagram of the study population 